\title{
Brain-Derived Neurotrophic Factor-Induced Gene Expression Reveals Novel Actions of VGF in Hippocampal Synaptic Plasticity
}

\author{
Janet Alder, ${ }^{1 \star}$ Smita Thakker-Varia, ${ }^{1 \star}$ Debra A. Bangasser, ${ }^{2}$ May Kuroiwa, ${ }^{1}$ Mark R. Plummer, ${ }^{3}$ Tracey J. Shors, ${ }^{2}$ and \\ Ira B. Black ${ }^{1}$ \\ ${ }^{1}$ Department of Neuroscience and Cell Biology, University of Medicine and Dentistry of New Jersey, Robert Wood Johnson Medical School, Piscataway, New \\ Jersey 08854-5635, ${ }^{2}$ Department of Psychology and Center for Collaborative Neuroscience, and ${ }^{3}$ Faculty of Arts and Sciences Division of Life Sciences, \\ Nelson Laboratories, Rutgers University, Piscataway, New Jersey 08854
}

Synaptic strengthening induced by brain-derived neurotrophic factor (BDNF) is associated with learning and is coupled to transcriptional activation. However, identification of the spectrum of genes associated with BDNF-induced synaptic plasticity and the correlation of expression with learning paradigms in vivo has not yet been studied. Transcriptional analysis of BDNF-induced synaptic strengthening in cultured hippocampal neurons revealed increased expression of the immediate early genes (IEGs), $c$-fos, early growth response gene 1 (EGR1), activity-regulated cytoskeletal-associated protein $(A r c)$ at $20 \mathrm{~min}$, and the secreted peptide $V G F$ (non-acronymic) protein precursor at $3 \mathrm{hr}$. The induced genes served as prototypes to decipher mechanisms of both BDNF-induced transcription and plasticity. BDNF-mediated gene expression was tyrosine kinase B and mitogen-activated protein kinase-dependent, as demonstrated by pharmacological studies. Single-cell transcriptional analysis of $A r c$ after whole-cell patch-clamp recordings indicated that increased gene expression correlated with enhancement of synaptic transmission by BDNF. Increased expression in vitro predicted elevations in vivo: VGF and the IEGs increased after trace eyeblink conditioning, a hippocampal-dependent learning paradigm. VGF protein was also upregulated by BDNF treatment and was expressed in a punctate manner in dissociated hippocampal neurons. Collectively, these findings suggested that the VGF neuropeptides may regulate synaptic function. We found a novel function for VGF by applying VGF peptides to neurons. C-terminal VGF peptides acutely increased synaptic charge in a dose-dependent manner, whereas $\mathrm{N}$-terminal peptide had no effect. These observations indicate that gene profiling in vitro can reveal new mechanisms of synaptic strengthening associated with learning and memory.

Key words: VGF; BDNF; synaptic plasticity; gene expression; hippocampus; learning

\section{Introduction}

Neurotrophin-induced modifications of synaptic strength have been associated with mechanisms of learning and memory (Chao, 2000; Tyler et al., 2002). However, the molecular genetics of brain-derived neurotrophic factor (BDNF)-induced plasticity have not been explored. Several studies have drawn links between BDNF, learning, transcription, and translation. For example, endogenous BDNF as well as transcriptional activation are necessary for the maintenance phase of long-term potentiation (LLTP) (Korte et al., 1998; Chen et al., 1999; Patterson et al., 2001).

Received Aug. 11, 2003; revised Sept. 22, 2003; accepted Sept. 28, 2003.

This work was funded by grants from the National Institute of Child Health and Human Development, National Alliance for Research on Schizophrenia and Depression, The Foundation of University of Medicine and Dentistry of New Jersey, and the New Jersey Commission for Science and Technology. We are grateful to $S$. Salton for providing VGF peptides. We thank C. Donahue and K. Kosik for RNA from trained rats and M. Borrero for technical help with VGF immunocytochemistry and Western blots.

*J.A. and S.T.-V. contributed equally to this work.

Correspondence should be addressed to Dr. Ira B. Black, Department of Neuroscience and Cell Biology, University of Medicine and Dentistry of New Jersey-Robert Wood Johnson Medical School, 675 Hoes Lane, Center for Advanced Biotechnology and Medicine, Piscataway, NJ 08854-5635. E-mail: black@cabm.rutgers.edu.

Copyright $\odot 2003$ Society for Neuroscience $\quad 0270-6474 / 03 / 2310800-09 \$ 15.00 / 0$
In addition, $\mathrm{BDNF}$ and tyrosine kinase $\mathrm{B}$ (trkB) may be required for the acquisition, consolidation, and recall of some types of information (Tyler et al., 2002; Yamada et al., 2002). Finally, $\mathrm{BDNF}$ alone is sufficient to trigger a form of long-term synaptic potentiation in vivo (BDNF-LTP) (Ying et al., 2002), the induction of which requires transcription (Messaoudi et al., 2002). The regulation and function of specific candidate genes in BDNFLTP, L-LTP, and learning paradigms (Hevroni et al., 1998; Guzowski et al., 2001; Ying et al., 2002) have been demonstrated. Although studies have used transcriptional profiling to define panels of genes associated with learning (Luo et al., 2001; Cavallaro et al., 2002; Donahue et al., 2002), the identification of a catalog of genes associated with BDNF-induced plasticity in vitro and the correlation of expression of these genes with learning in vivo has not yet been studied.

The regulated genes identified by transcriptional profiling serve as powerful tools to decipher mechanisms of both BDNFinduced transcription and plasticity. These genes may be used as prototypes to explore whether the effect of BDNF on synaptic strengthening in individual hippocampal cells is reflected in transcriptional alterations. We and others have reported that BDNF 
elicits differential electrophysiological responses in individual neurons (Levine et al., 1996; Gottschalk et al., 1998; Lessmann and Heumann, 1998; Schinder et al., 2000; Thakker-Varia et al., 2001). This phenomenon has been attributed to target specificity (Schinder et al., 2000) and/or initial synaptic strength (Gottschalk et al., 1998; Lessmann and Heumann, 1998; Berninger et al., 1999); however, these studies did not address the molecular mechanisms associated with distinctive single-cell synaptic responses to BDNF. Moreover, although the signal transduction pathways required for BDNF-modulated synaptic plasticity have been examined (Gottschalk et al., 1999; Minichiello et al., 2002; Mizuno et al., 2003), the requirement for various kinase pathways in BDNF-induced transcription remains to be determined.

Increased transcription during the processes of learning in vivo suggests that gene products may be involved in the formation of new memories. Transcriptional analysis after different learning tasks has revealed regulation of genes previously implicated in synaptic function (Guzowski et al., 2001; Luo et al., 2001; Cavallaro et al., 2002; Donahue et al., 2002). In the present set of experiments, we evaluated gene transcription in response to learning a classically conditioning eyeblink response. This task is well suited for this purpose, because transcription in animals that learn the task can be directly compared to that in animals that are exposed to the same stimuli but do not learn a positive association (Donahue et al., 2002). Memory formation is regulated by neuronal plastic changes, which may be mediated by the protein products of induced genes.

There are only a few studies delineating functional roles for neuronal genes identified by transcriptional profiling (Huh et al., 2000). We previously used differential display in conjunction with electrophysiological analysis using gene-targeted mice to identify a requirement for Rab3A, a synaptic vesicle-trafficking protein, in BDNF-induced plasticity (Thakker-Varia et al., 2001). Despite the limited screen performed in that study, we were able to define a functional role for one of the identified genes and begin to define molecular pathways in BDNF-mediated synaptic strengthening.

To further elucidate the molecular bases of BDNF-induced synaptic strengthening (Chao, 2000; Thoenen, 2000; Poo, 2001; Lu, 2003), we now use cDNA microarrays in combination with patch clamp. We detected upregulation of several IEGs, including $A r c$, as well as a number of genes not yet associated with BDNFinduced plasticity, such as the secreted polypeptide VGF. Arc expression correlated with single-cell electrophysiological responses to BDNF. Moreover, transcriptional changes of Arc as well as VGF elicited by BDNF in vitro predicted increases with learning paradigms in vivo. Furthermore, the novel finding that exogenous application of VGF peptides induced synaptic enhancement suggests the importance of VGF and Arc in learning and memory processes.

\section{Materials and Methods}

Cell culture preparation. Time-mated pregnant rats were killed by $\mathrm{CO}_{2}$ asphyxiation in accordance with institutional guidelines for care and use of animals. Fetuses were removed by caesarean section and transferred to a sterile Petri dish with PBS. Fetal hippocampi were dissected from surrounding brain tissue, and meninges were completely removed. Lowdensity cultures of dissociated embryonic day 18 rat hippocampi (Sprague Dawley; Hilltop Laboratories, Scottsdale, PA) were prepared as described (Thakker-Varia et al., 2001). Briefly, pooled tissue from each litter was mechanically dissociated in nutrient medium containing $7.5 \%$ fetal bovine serum and plated on poly-D-lysine-coated culture dishes at
350,000 cells per dish. Cultures were maintained in serum-free medium (Thakker-Varia et al., 2001) for 10-14 d and contained pure neurons.

Electrophysiological recordings. Whole-cell patch-clamp recordings were performed after 10-14 d in culture. Currents were recorded with an Axoclamp 200 amplifier, digitized at $2.5 \mathrm{kHz}$ with an INDEC IDA 15125 interface, filtered at $5 \mathrm{kHz}$, and stored. Recording parameters and stimulus protocols were controlled by custom software written with Borland $\mathrm{C}++$ that utilizes device driver libraries supplied by INDEC. Data analysis programs were written with Microsoft (Seattle, WA) Visual Basic. The external bath solution for voltage-clamp recordings was (in $\mathrm{mm}$ ): $1.67 \mathrm{CaCl}_{2}, 1 \mathrm{MgCl}_{2}, 5.36 \mathrm{KCl}, 137 \mathrm{NaCl}, 17$ glucose, 10 HEPES, and 20 sucrose [neuron recording solution (NRS)]. The pipette solution contained (in mM): 105 Cs-methanesulfonate, $17.5 \mathrm{CsCl}, 10 \mathrm{HEPES}, 0.2$ EGTA, $8 \mathrm{NaCl}$, $2 \mathrm{Mg}$-ATP, $2 \mathrm{Na}$-ATP, $0.3 \mathrm{Na}$-GTP, 20 phosphocreatinine, and $50 \mathrm{U} / \mathrm{ml}$ creatinine phosphokinase. All recordings were made at room temperature. The typical range of pipette resistance was 3-5 M 2 . Cell capacitance was $10-20 \mathrm{pF}$, and access resistance was 7-20 M $\Omega$. Vacuum perfusion system contained BDNF (50 ng/ml; Preprotech, Princeton, NJ), NRS as a control, or VGF peptides $(0.1 \mu \mathrm{M}=740 \mathrm{ng} / \mathrm{ml})$. Three synthetic rat VGF peptides (gift from S. Salton, Mount Sinai School of Medicine, New York, NY) were used: LEGS-28 amino acids (aa) 285-312 (amidated), TLQP-62 aa 556-617, and AQEE-30 aa 588617. Each data point represents a cell from a separate dish, and at least three different platings were used for each condition.

Data analysis. Data were analyzed by integrating the synaptic currents for each sweep (synaptic charge). The charge measurements for all sweeps in a $1 \mathrm{~min}$ period were averaged (binned). Baseline is considered the average synaptic charge during the $2 \mathrm{~min}$ period ( -2 to $0 \mathrm{~min}$ ) in NRS immediately before BDNF or VGF application. Fold increases were then determined by dividing the synaptic charge during BDNF or VGF exposure by the baseline. Recordings were rejected if either the $0-5 \mathrm{~min}$ binned time period or 5-10 min binned time period after switching during BDNF or VGF exposure was $2 \times$ SEM below baseline, indicating "run down."

Microarray. Total cellular RNA was prepared from neuronal cultures treated with either BDNF $(50 \mathrm{ng} / \mathrm{ml}$ ) or vehicle (water) for either $20 \mathrm{~min}$ or $3 \mathrm{hr}$ by the guanidine isothiocyanate method and $\mathrm{CsCl}$ gradients (Thakker-Varia et al., 2001). DNase-treated total RNA was prepared from neurotrophin or control hippocampal cultures and reversetranscribed using gene-specific primers. Radioactively labeled probes were then hybridized to Atlas Arrays Rat 1.2 (Clontech, Palo Alto, CA) according to manufacturer's protocol. Arrays were performed in duplicate with an independent source of RNA. The hybridization pattern was analyzed on a phosphorimager and normalized to the global hybridization signal. Analysis was performed by Clontech (Palo Alto, CA) using 1.7-fold change as criteria for significance. All transcriptional changes were confirmed by real time reverse transcription PCR (RT-PCR) on independent samples (see below).

Real time RT-PCR. One hundred microliters of cDNA were prepared from $2 \mu \mathrm{g}$ of control or BDNF-treated RNA using random primers and Superscript II reverse transcriptase. Twenty-five microliter PCR reactions were then performed using gene-specific primers designed by Primer Express software and TaqMan MGB probes (7000 Sequence Detection System; Applied Biosystems Foster City, CA). Duplicate wells were included for each condition and primer pair. Primers specific to the housekeeping gene glyceraldehyde-3-phosphate dehydrogenase $(G A P D H)$ were used as an internal control. Data analysis was performed according to the protocol provided by Applied Biosystems.

Single-cell antisense RNA amplification. After whole-cell patch-clamp recording for $\sim 30 \mathrm{~min}$, the cellular contents were aspirated into the patch pipette with a small amount of suction. cDNA synthesis was performed in vitro using the MessageAmp antisense RNA (aRNA) kit (Ambion, Austin, TX). Two rounds of aRNA synthesis were performed resulting in a one million-fold amplification of the original material (Phillips and Eberwine, 1996). Twenty microliters of cDNA were synthesized from all of the aRNA, and $2 \mu \mathrm{l}$ was used as a template in real-time PCR for Arc and GAPDH gene expression, as described above, with the exception that 65 amplification cycles were used.

Pharmacological treatments. Hippocampal neurons were preincubated 
Table 1. Genes induced by BDNF treatment

\begin{tabular}{|c|c|c|c|c|c|}
\hline Clone number & Gene/Protein name & Abbreviation & Accession number & Fold change & NGF \\
\hline \multicolumn{6}{|l|}{$20 \mathrm{~min}$ BDNF } \\
\hline $\mathrm{A} 10 \mathrm{~g}$ & c-fos proto-oncogene & $c-f o s$ & X06769 & $2.2 \pm 0.4$ & UC \\
\hline E06d & Early growth response protein 1 & EGR1 & M18416 & $4.3 \pm 0.8$ & $U C$ \\
\hline F13I & Activity-regulated cytoskeletal-associated & Arc & U19866 & $13.7 \pm 5.0$ & UC \\
\hline \multicolumn{6}{|l|}{$3 \mathrm{hr}$ BDNF } \\
\hline A05d & fos-related antigen 2 & Fra2 & U18913 & $2.3 \pm 0.4$ & down \\
\hline A07a & G1/S-specific cyclin D1 & cyclin D1 & D14014 & $3.3 \pm 0.5$ & UC \\
\hline $\mathrm{A} 10 \mathrm{~g}$ & c-fos proto-oncogene & $c-f o s$ & X067679 & $2.3 \pm 0.4$ & UC \\
\hline B05d & Voltage-gated potassium channel protein & $K v+1.1$ & M26161 & $1.4 \pm 0.1$ & $U C$ \\
\hline B06j & Sodium channel, beta 1 subunit & $\mathrm{Na}+\mathrm{ch}$ & M91808 & $1.8 \pm 0.2$ & $U C$ \\
\hline B13n & Secretogranin II precursor & Sgll & M93669 & $5.1 \pm 1.2$ & UC \\
\hline D04i & Somatostatin receptor 4 & SSTR4 & U04738 & $1.7 \pm 0.1$ & down \\
\hline D11k & Transmembrane receptor UNC5 homolog & UNC5H2 & U87306 & $2.2 \pm 0.6^{*}$ & UC \\
\hline D13l & Neuropeptide Y precursor & NPY & M20373 & $1.9 \pm 0.3^{*}$ & down \\
\hline D14f & VGF protein precursor & VGF & M60525 & $6.5 \pm 1.6$ & $U C$ \\
\hline E06d & Early growth response protein 1 & EGR1 & M18416 & $6.6 \pm 0.9$ & $U C$ \\
\hline E12f & Protein-tyrosine phosphatase 1B & PTP1B & M33962 & $1.4 \pm 0.1$ & $U C$ \\
\hline F13I & Activity-regulated cytoskeletal-associated & Arc & U19866 & $34.8 \pm 4.3$ & UC \\
\hline
\end{tabular}

Two independent Atlas arrays were performed using RNA from hippocampal cells treated for either $20 \mathrm{~min}$ or $3 \mathrm{hr}$ with BDNF ( $50 \mathrm{ng} / \mathrm{ml}$ ) and control sister cultures. The criteria for selection from the array was a $>1.7$-fold change. Real time RT-PCR validated the induction of 16 genes (average $\pm \mathrm{SE} ; n=6 ;{ }^{*} n=5 ; p<0.05 ; t$ test). NGF was used as a neurotrophin control that does not induce plasticity. All gene changes appear to be specific to BDNF. UC, Unchanged; down, decreased.

with inhibitors for 30 min before BDNF treatment (20 min for IEGs and $3 \mathrm{hr}$ for VGF). All inhibitors were purchased from Calbiochem (San

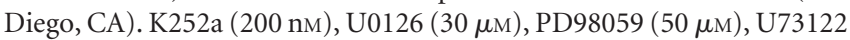
$(10 \mu \mathrm{M}), \mathrm{KN} 93(10 \mu \mathrm{M}), \mathrm{LY} 294002(30 \mu \mathrm{M}), \mathrm{H}-89(1 \mu \mathrm{M})$, and chelerythrine chloride (CC; $1 \mu \mathrm{M})$. Pharmacological inhibitors were tested for efficacy by assaying downregulation of endogenous kinase phosphorylation in cell culture using Western blot (Y. Z. Du and C. F. Dreyfus, personal communication). Each experiment consisted of four groups: vehicle, inhibitor alone, BDNF, and BDNF plus inhibitor. After inhibitor and BDNF treatments, RNA was isolated and subjected to real time RTPCR as described above.

SDS-PAGE and Western blot analysis. BDNF-treated and untreated hippocampal neurons plated in equal numbers were solubilized in lysis buffer (20 mM Tris, pH 8, 0.5\% Triton X-100, 0.5\% SDS, protease inhibitor tablet, $1 \mathrm{~mm}$ PMSF, and $0.5 \mathrm{~mm}$ vanadate). The protein content was determined with the BCA protein assay kit (Pierce, Rockford, IL). Samples containing equal amounts of protein were denatured in Laemmli's sample buffer (containing $\beta$-mercaptoethanol) for $5 \mathrm{~min}$ and subjected to $8-12 \%$ gradient SDS-PAGE (Suen et al., 1997). The proteins were transferred to polyvinylidene difluoride (PVDF) membranes (Millipore, Bedford, MA) that were blocked for $1 \mathrm{hr}$ with a $5 \%$ solution of dry milk powder plus $5 \%$ normal serum in $0.1 \%$ Tween 20 and PBS. The PVDF membranes were incubated with goat anti-R15 VGF (1:500) (Santa Cruz Biotechnology, Santa Cruz, CA) or goat anti-actin (1:500) (Santa Cruz) with $1 \%$ bovine serum albumin overnight at $4^{\circ} \mathrm{C}$. Membranes were washed, followed by $1 \mathrm{hr}$ incubation with donkey anti-goat horseradish peroxidase-conjugated IgG (1:5000) at room temperature. The immunopositive bands were visualized by chemiluminescence using the ECL detection kit (NEN, Boston, MA), quantitated on a Bio-Rad (Hercules, CA) Gel Doc and normalized to actin.

Immunocytochemistry. Cultures were fixed in $4 \%$ paraformaldehyde. A blocking step was performed in 30\% normal horse serum in PBS containing $0.3 \%$ Triton X-100. VGF antibody (R15) (1:100) (Santa Cruz) was applied overnight at $4^{\circ} \mathrm{C}$. Cultures were incubated for $1 \mathrm{hr}$ at room temperature with Alexa Flour 594 donkey anti-goat secondary (1:500) (Molecular Probes, Eugene, OR) and visualized on a Leica (Nussloch, Germany) fluorescent microscope (DMIRB).

Trace eyeblink conditioning. Classical eyeblink conditioning methods followed published procedures (Donahue et al., 2002; Leuner et al., 2003). Generally, an auditory stimulus is associated with stimulation to the eyelid. After repeated pairings, the animals respond to the auditory stimulus in anticipation of the eyelid stimulation. When a temporal gap is placed between the auditory stimulus and the eyelid stimulation, learning becomes hippocampal-dependent (Beylin et al., 2001). Specifically, groups of rats were exposed to 200 trials per day for $4 \mathrm{~d}$ for a total of 800 trials. The rats were trained with a trace paradigm in which a $250 \mathrm{msec} 82$ $\mathrm{dB}$ burst of white noise (CS) was separated from a $100 \mathrm{msec}, 0.7 \mathrm{~mA}$ periorbital shock (US) by a $500 \mathrm{msec}$ trace interval. The intertrial interval was $25 \pm 5 \mathrm{sec}$. During unpaired training, rats received the same number of CS and US exposures, but presented in an explicitly unpaired manner. Naive rats did not receive stimulus exposure. Rats were anesthetized with Nembutal $(50 \mathrm{mg} / \mathrm{kg})$ and decapitated immediately after training. The hippocampus was dissected and frozen on dry ice. RNA was prepared from the tissue and subjected to real time RT-PCR as described above.

\section{Results}

\section{BDNF induces multiple classes of genes}

To begin defining the molecular genetics underlying plasticity evoked by BDNF, expression profiling was performed using cDNA microarrays on a population of hippocampal cells in vitro. Synaptic effects of BDNF are observed within 20 min of exposure (Levine et al., 1996; Lessmann and Heumann, 1998; Schinder et al., 2000). Our previous studies using a $3 \mathrm{hr}$ BDNF treatment identified several genes with roles in synaptic plasticity (ThakkerVaria et al., 2001). Consequently, to reveal immediate as well as downstream transcriptional changes, both acute $(20 \mathrm{~min})$ and longer treatment $(3 \mathrm{hr})$ with BDNF were used. Approximately $8 \%$ of the arrayed cDNAs exhibited differential expression at 20 $\mathrm{min}$ and $3 \mathrm{hr}$. Genes potentially involved in synaptic plasticity and exhibiting an increase of 1.7-fold over control were selected for confirmation by real time RT-PCR (Table 1). Of those genes selected, the regulation of $7 \%$ at $20 \mathrm{~min}$ and $40 \%$ at $3 \mathrm{hr}$ were validated, although the magnitude of regulation was not always identical to that observed on the microarray. To focus on genes specifically involved in plasticity, a related neurotrophin, nerve growth factor (NGF), not inducing synaptic plasticity (Levine et al., 1996), served as a control. NGF did not affect transcription of most genes (Table 1), suggesting the specificity of BDNF.

Genes upregulated by BDNF can be categorized into different functional classes. At $20 \mathrm{~min}$, IEGs were upregulated, whereas at 3 $\mathrm{hr}$, in addition to those same IEGs, channel proteins, receptors, and neuropeptides exhibited increases. Two genes were of particular interest because of their association with synaptic plasticity: Arc/Arg 3.1, an effector IEG and VGF, a secreted polypeptide located in large dense core vesicles (Salton et al., 2000). Arc, the 
A

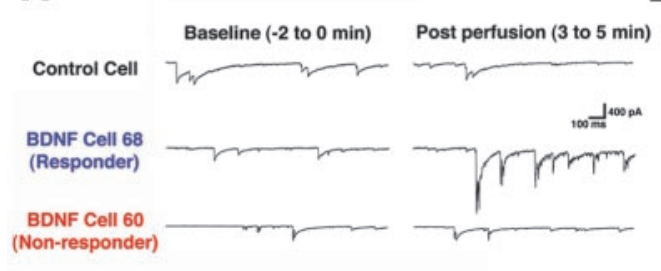

B

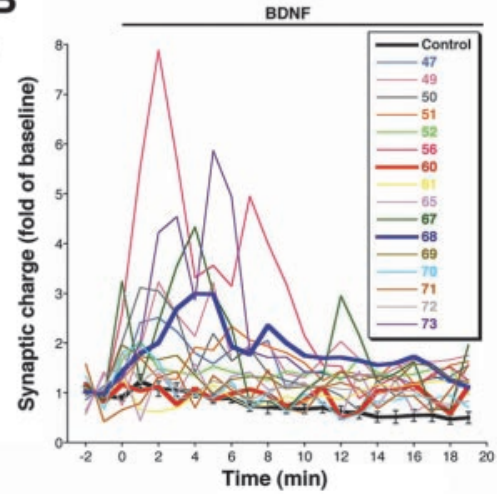

Figure 1. Hippocampal pyramidal-like neurons exhibit differential acute responses to BDNF. $A$, Example whole-cell voltageclamp recordings $\left(V_{\text {hold }}=-60 \mathrm{mV}\right.$ ) from three individual hippocampal neurons during baseline recordings (NRS perfusion, -2 to $0 \mathrm{~min}$ ) and 3-5 min after switching perfusion to either NRS (control) or BDNF $(50 \mathrm{ng} / \mathrm{ml}$ ). Note that cell 68 (blue) responds to BDNF, whereas cell 60 (red) does not. $B$, Synaptic charge in 1 min bins over a 20 min recording period for control neurons (average $\pm \mathrm{SE} ; n=7$ ) (thick black line), BDNF cell 68 (thick blue line), and BDNF cell 60 (thick red line) as well as all other individual neurons (all other colors; $n=16$ ) exposed to BDNF. The differential response to BDNF is demonstrated. Recordings were obtained from multiple platings.

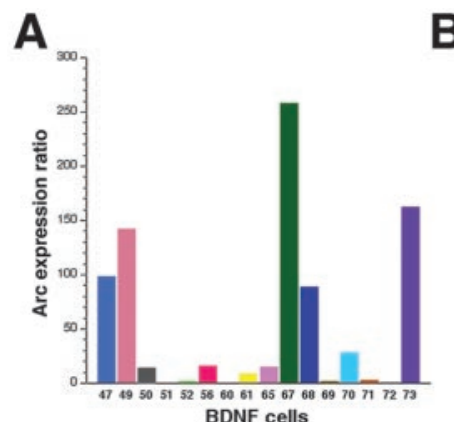

BDNF cells
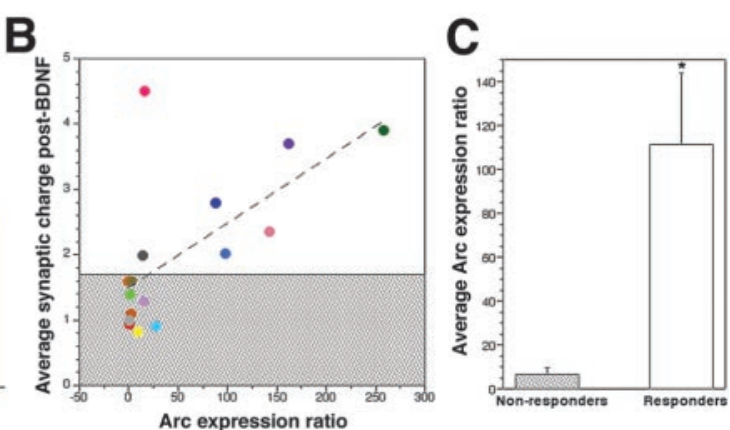

Figure 2. Arc expression and synaptic response to BDNF correlate at the single-cell level. $A$, Arc expression in individual BDNF-treated cells shown in Figure 1 after electrophysiological recordings and aRNA amplification. Arc transcription was measured by real time RT-PCR and then normalized to internal GAPDH levels. The data are then expressed as a ratio of the average Arc expression in the seven control cells (expression ratio). Colors for individual cells correspond to those in Figure 1. A large variability of Arc gene levels is apparent among the individual BDNF-treated cells. B, Correlation of Arc expression ratios to the synaptic BDNF responses of individual cells. Average synaptic charge 3-5 min after BDNF exposure expressed as a fold increase over baseline $(-2$ to 0 min) (Fig. 1) was plotted against Arc expression ratios shown in A. Each circle represents one BDNF-treated cell of corresponding color. Dashed line depicts a correlation coefficient indicating significance $(r=0.66 ; p<0.005)$. The data are categorized into two groups of BDNF-treated cells: a cluster of non-responders (shaded) and a spread of responders (clear). C, Average Arcexpression ratios of the two populations of cells defined in $B$. Responders (white bar) show a significantly higher fold increase in Arc expression (average $\pm \mathrm{SE} ; n=7$ ) compared with non-responders (shaded bar) (average $\pm \mathrm{SE} ; n=9)\left({ }^{*} p<0.05 ; t\right.$ test).

ter 3 hr BDNF exposure (Thakker-Varia et al., 2001). Whole-cell patch-clamp recordings from 16 cells verified a range of responses from 0.8 - to 4.5 -fold increases in synaptic charge after 20 min BDNF exposure (Fig. 1). Average synaptic charge for control cells not exposed to $\operatorname{BDNF}(n=7)$ exhibited no change in synaptic charge during the length of the recording. A group of BDNF-treated cells presenting a minimal synaptic response was apparent (Fig. 2B). We categorized this group as non-responders, and the other cells showing a greater response to BDNF were classified as responders. To determine whether cellular basal activity influences the response to BDNF, the average synaptic charge before BDNF of the responders $(36.3 \pm 13.1 \mathrm{pC} ; n=7)$ versus the nonresponders $(23.2 \pm 6.6 \mathrm{pC} ; n=9)$ was compared. There was no statistical difference in the populations $(p>0.05 ; t$ test $)$ examined between initial synaptic strength and responsiveness to BDNF, suggesting that this parameter is not responsible for the differential response.

The molecular genetics of differential synaptic responses to BDNF was explored by performing single-cell transcriptional analysis after whole-cell patch clamp. Arc was selected as a prototype because of its acute and robust induction by BDNF in a population of hippocampal neurons ( $\mathrm{Ta}$ ble 1). To assess the relationship between differential electrophysiological responses to BDNF (Fig. 1) and Arc gene expression, individual cells were harvested after electrophysiological recordings. Figure $2 \mathrm{~A}$ represents the $A r c$ expression in cells exposed to BDNF relative to the average Arc expression in cells not exposed to BDNF (control) as assessed by real time RT-PCR (expression ratio). A range of Arc expression levels was apparent within the group of 16 BDNF-treated cells, indicating transcriptional differences among pyramidal-

most highly regulated gene at both $20 \mathrm{~min}$ and $3 \mathrm{hr}$, has been implicated in BDNF-induced plasticity and LTP (Guzowski et al., 2000; Messaoudi et al., 2002; Steward and Worley, 2002; Ying et al., 2002). VGF, previously cloned from plate $V$ of a NGF-induced PC12 cDNA library (Salton et al., 2000), in contrast, was only increased at $3 \mathrm{hr}$ and has not been associated with BDNF-induced synaptic efficacy. These two genes were used in subsequent experiments as indices of synaptic efficacy and prototypes for functional studies.

\section{Arc expression and synaptic response to BDNF correlate at the single-cell level}

To examine the relationship between synaptic response and gene expression, we examined the electrophysiological response of the individual cells to BDNF in conjunction with single-cell RT-PCR for Arc. Previous studies revealed a range ofsynaptic activities within a population of pyramidal-like hippocampal neurons af- like hippocampal cells.

The relationship between the synaptic response to BDNF and transcriptional changes was examined. Synaptic charge was plotted against Arc expression ratios for individual cells (Fig. $2 B$ ). A linear correlation $(r=0.66 ; p<0.005)$ is evident, as depicted by the dashed line, suggesting a direct relationship between synaptic plasticity and gene expression. Alternatively, the data can be viewed as representing two sets of BDNF-treated cells: a cluster of non-responders (shaded area) and a spread of responders (clear area). Classification of cells into electrophysiologic responders or non-responders reveals a striking difference in Arc expression ratios (Fig. 2C). Responders exhibit a 17-fold increase in Arc expression relative to the non-responders ( $p<0.05 ; t$ test). In aggregate, this is the first demonstration that the magnitude of BDNF-induced synaptic plasticity is reflected in gene expression in individual hippocampal cells. Enhanced transcription after neurotrophin exposure may represent a mechanism for long- 
Table 2. Effect of inhibitors on BDNF-induced gene transcription

\begin{tabular}{|c|c|c|c|c|c|c|c|c|}
\hline Gene & $\begin{array}{l}\text { K252a } \\
\text { (tyr kinase) }\end{array}$ & $\begin{array}{l}\text { U0126 } \\
\text { (MEK) }\end{array}$ & $\begin{array}{l}\text { PD98059 } \\
\text { (MEK) }\end{array}$ & $\begin{array}{l}\text { U73122 } \\
\text { (PLC- } \gamma)\end{array}$ & $\begin{array}{l}\text { LY294002 } \\
\text { (PI3 kinase) }\end{array}$ & $\begin{array}{l}\text { KN93 } \\
\text { (CaMKII) }\end{array}$ & $\begin{array}{l}\text { H-89 } \\
\text { (PKA) }\end{array}$ & $\begin{array}{l}\mathrm{CC} \\
\text { (PKC) }\end{array}$ \\
\hline \multicolumn{9}{|l|}{$20 \mathrm{~min}$} \\
\hline$c-f o s$ & Blocked & Blocked & Blocked & Blocked & UC & Blocked & UC & UC \\
\hline EGR1 & Blocked & Blocked & Blocked & UC & UC & Blocked & UC & UC \\
\hline Arc & Blocked & Blocked & Blocked & Blocked & $U C$ & Blocked & UC & UC \\
\hline \multicolumn{9}{|l|}{$3 \mathrm{hr}$} \\
\hline VGF & Blocked & Blocked & Blocked & Blocked & $U C$ & Blocked & UC & UC \\
\hline
\end{tabular}

The regulation of BDNF-induced genes in the presence of inhibitors was determined by real time RT-PCR. Neurons were preincubated with inhibitors for 30 min before BDNF treatment ( 20 min for IEGs and 3 hr for $V G F$. All samples were firs normalized to GAPDH and then represented as a ratio of vehicle controls. One group of cells in each experiment was treated with inhibitor alone and showed no significant change in expression relative to vehicle control. "Blocked" indicates significant reduction of transcription in the presence of inhibitor plus BDNF relative to BDNF alone ( $p<0.05 ;$ ANOVA; $n=3$ ). UC (unchanged) indicates no significant effector of inhibitor on transcription. K242a, U0126, PD98059, U73122, and KN93 blocked BDNF-induced transcription of the IEGs and VGF, whereas LY294002, H-89, and CC did not affect gene expression.

term maintenance of increased synaptic activity, as has been shown for L-LTP (Nguyen et al., 1994). It is therefore important to elucidate the signaling pathways mediating BDNF-induced transcription.

\section{Transcriptional regulation of IEGs and VGF by BDNF requires trkB and MAP kinases}

Signaling cascades involved in BDNF-mediated synaptic strengthening include mitogen-activated protein (MAP) kinase, phospholipase C- $\gamma$ (PLC $\gamma$ ), and phosphoinositide-3 kinase (PI3K) (Gottschalk et al., 1999; Minichiello et al., 2002; Ying et al., 2002; Mizuno et al., 2003). To determine whether these transduction pathways are also required for BDNF transcriptional regulation of IEGs at $20 \mathrm{~min}$ and for $V G F$ at $3 \mathrm{hr}$, pharmacological inhibitors were used (Table 2). Each experiment consisted of four groups: vehicle, inhibitor alone, BDNF, and BDNF plus inhibitor. Data were normalized to GAPDH and expressed as a fold change of vehicle control. Inhibitor alone showed no significant effect on transcription. K252a blocked the BDNF effect, suggesting that transcription results from trkB stimulation. To examine MAP kinases, two independent inhibitors were used, U0126 and PD98059. Pretreatment with either inhibitor blocked BDNFinduced transcription, suggesting a requirement for MEKmediated phosphorylation of MAP kinase. The PLC $\gamma$ inhibitor (U73122) and the $\mathrm{Ca}^{2+}$-calmodulin-dependent protein kinase (CaMK) inhibitor (KN93) blocked transcription of most IEGs and $V G F$ (Table 2). In contrast, a number of signaling pathways were not involved in BDNF-induced transcription, shown by the lack of effect of inhibitors of PI3K (LY294002), protein kinase A (PKA), (H-89), and protein kinase C (PKC) (CC) (Table 2). These results suggest that BDNF-mediated transcription at both $20 \mathrm{~min}$ and $3 \mathrm{hr}$ is dependent on the CaMK and PLC $\gamma$ pathways in addition to trkB and MAP kinase.

\section{Trace eyeblink conditioning induces expression of VGF and IEGs}

To determine whether $V G F$ and other transcripts induced by BDNF in vitro predict alterations in mRNA in vivo, gene expression was examined after training on a hippocampal-dependent learning task. Rats were exposed to 800 trials of either paired or unpaired trace eyeblink conditioning and killed $24 \mathrm{hr}$ later. Eyeblinks were assessed by eyelid electromyography, and those occurring during the 500 msec trace interval were considered conditioned responses (CRs). Rats exposed to paired stimuli exhibited $60 \%( \pm 4)$ CRs, whereas those exposed to the same number of stimuli presented in an explicitly unpaired manner exhibited $11 \%( \pm 1)$ CRs, a percentage similar to that of spontaneous blinks (10\%). A $t$ test for independent samples on the number of conditioned responses revealed that the difference was

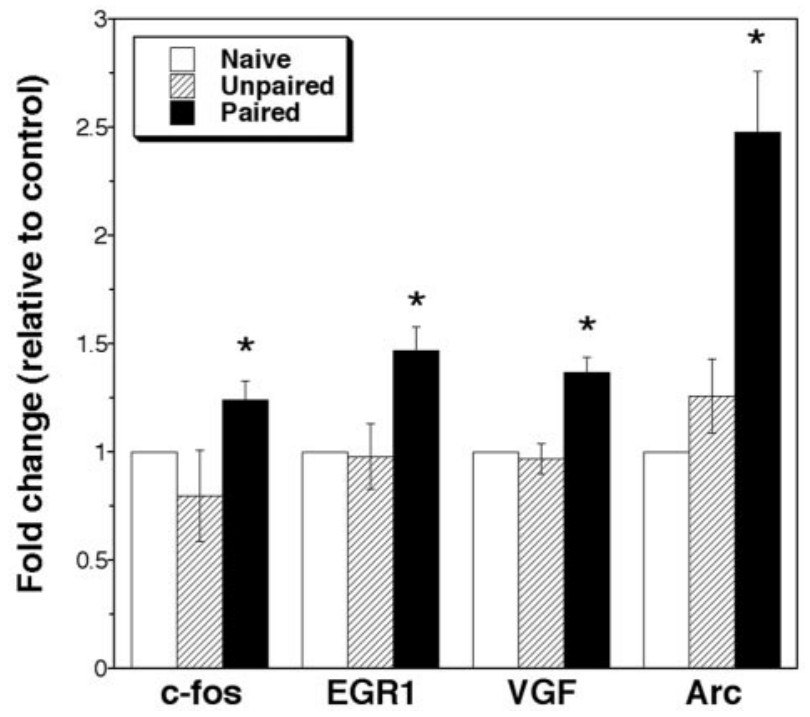

Figure 3. VGFas well as IEGs are upregulated after training with trace eyeblink conditioning. RNA was obtained from the hippocampi of naive rats, unpaired controls, and trace-conditioned rats after 800 trials ( 200 trials per day for $4 \mathrm{~d}$ ) of paired stimuli and subjected to real time RT-PCR for a number of genes identified by microarray. Bars represent average gene expression $\pm \mathrm{SE}$ for naive rats ( $n=5$; white bar), unpaired controls ( $n=3$; stippled bar), and trace conditioning ( $n=8$; black bar). All data are normalized to internal GAPDH levels and then expressed as a fold change over the amount of mRNA in naive (control) rats. A number of IEGs including c-fos, EGRI, and Arc were upregulated after trace conditioning. In addition the secreted polypeptide VGF was increased after paired trace conditioning. *Indicates paired samples are significantly different from unpaired and naive control samples ( $p<0.05$; ANOVA).

significant at $p<0.0001$. VGF transcripts were significantly upregulated in the hippocampus after paired but not unpaired eyeblink conditioning or naive controls ( $p<0.05$; ANOVA) (Fig. 3). Several of the IEGs including $c$-fos, EGR1, and Arc were also induced by exposure to the paired stimuli ( $p<0.05$; ANOVA) (Fig. 3). These changes were specific and selective, because other genes induced by BDNF in vitro were not upregulated by trace eyeblink conditioning; $\mathrm{Na}^{+}$channel, somatostatin receptor 4 (SSTR4), and transmembrane receptor UNC homolog 2 (UNC5H2) were not significantly enhanced after paired eyeblink training (data not shown). These results indicate that BDNFinduced transcriptional alterations of VGF and IEGs in vitro predict changes in vivo in the hippocampus during memory formation. Consequently, the neuropeptide VGF is a potential candidate for regulation of synaptic function associated with learning and memory.

\section{VGF peptides acutely enhance synaptic activity}

The products of the genes induced after learning may regulate neuronal plasticity at the single-cell level. VGF, like BDNF, is a 


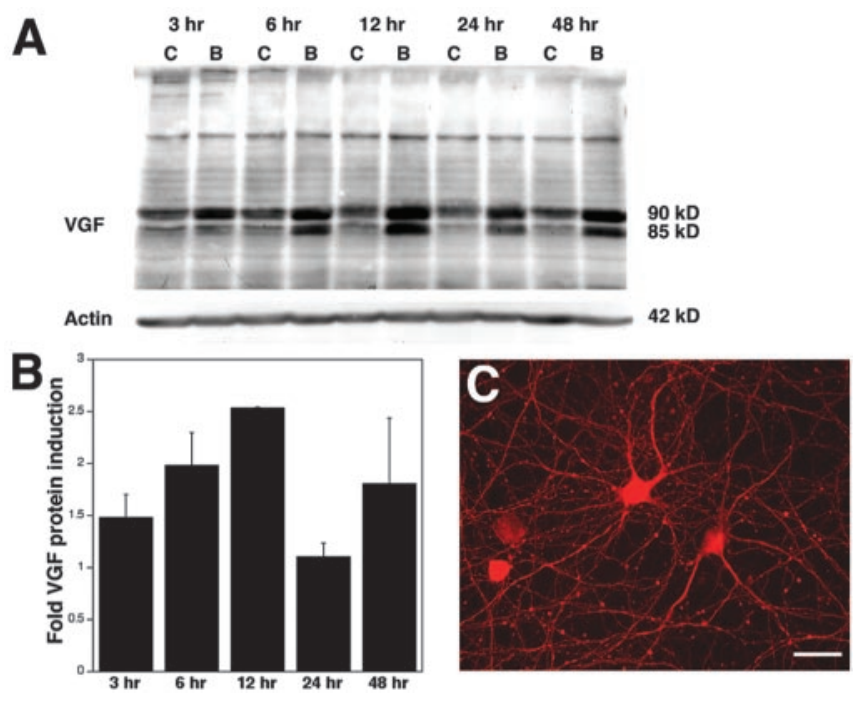

Figure 4. BDNF regulates the translation of the secreted polypeptide VGF. $A$, Western blot analysis of VGF expression after BDNF exposure $(50 \mathrm{ng} / \mathrm{ml})$. VGF protein appears as a doublet at 90 and $85 \mathrm{kDa}$. A minor high molecular weight species is detectable. $B$, Quantitation of VGF protein levels indicates that VGF is upregulated by $3 \mathrm{hr}$ and is maximal at $12 \mathrm{hr}$. Bars represent average VGF expression ( $\pm \mathrm{SE} ; n=2$ ). Actin is used as an internal control for protein levels, and data are expressed as fold of VGF expression in untreated sister cultures. C, Immunocytochemical localization of VGF in dissociated hippocampal neurons $12 \mathrm{~d}$ in vitro. Both the soma and the neurites of the pyramidal-like neurons are positive for VGF expression. Scale bar, $50 \mu \mathrm{m}$.

secreted polypeptide and thus may be relevant for intercellular communication. We therefore investigated a functional role for VGF in synaptic plasticity. First, to determine whether BDNFinduced transcriptional changes of $V G F$ are accompanied by translational alterations, VGF levels were examined after BDNF treatment by Western blot analysis (Fig. 4A,B). VGF, migrating as a doublet with an apparent molecular weight of $85-90 \mathrm{kDa}$, increased by 1.5 -fold within $3 \mathrm{hr}$ of BDNF exposure. VGF protein levels were maximal at $12 \mathrm{hr}$ and remained elevated for $48 \mathrm{hr}$ of continuous BDNF application. Consequently, increased VGF mRNA levels after 3 hr BDNF treatment are associated with enhanced protein translation. VGF protein processing occurs in both neurons and neuroendocrine cells (Trani et al., 1995; Salton et al., 2000). We examined the localization of VGF in dissociated hippocampal cultures using a C-terminal antibody (R-15), which labeled both the soma and neurites (Fig. 4C), indicating translocation to the neuronal processes. The expression of VGF was similar to that previously observed in primary neuronal cultures (Benson and Salton, 1996) and was punctate in appearance. This pattern is consistent with the regulated release of VGF from large dense core vesicles (Trani et al., 1995; Benson and Salton, 1996; Possenti et al., 1999) and suggests a physiological role for the peptide.

To explore a functional role for VGF in neuromodulation, whole-cell patch-clamp recordings on hippocampal cells were performed in the presence of synthetic VGF peptides. The electrophysiological profile of VGF peptides on hippocampal cells was similar to that observed for BDNF (Levine et al., 1996). Increased synaptic charge was observed within 5 min of perfusion of peptides derived from the C-terminal portion of VGF (TLQP62) (see Materials and Methods), whereas no change in synaptic charge resulted from application of peptide from the $\mathrm{N}$-terminal region (LEGS-28) (Fig. 5A,B). Enhanced synaptic activity was maintained for up to $25 \mathrm{~min}$ in the continued presence of TLQP-62 (Fig. 5B). As an independent confirmation of the effect

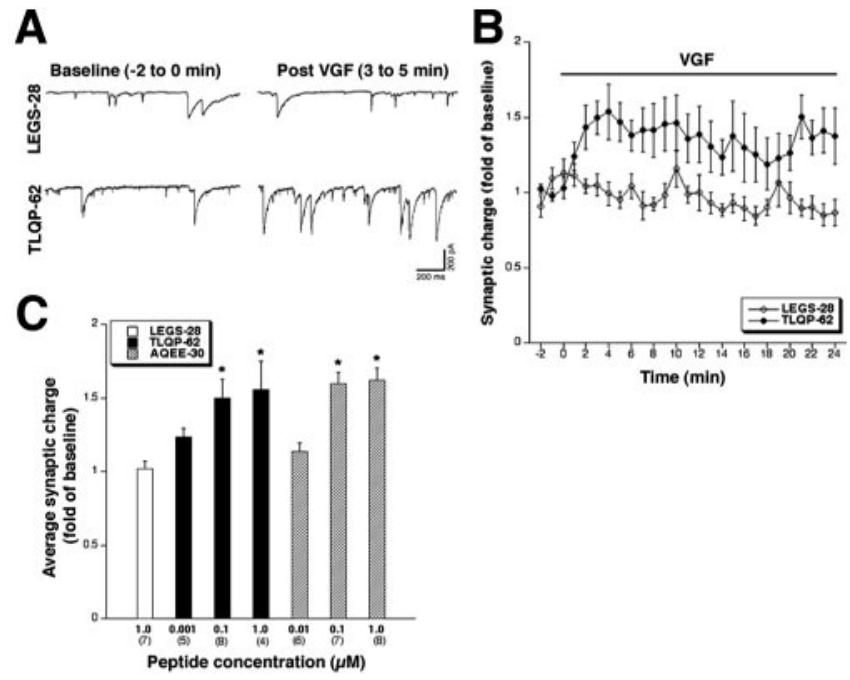

Figure 5. Exogenous VGF peptides acutely enhance synaptic activity. $A$, Representative traces of whole-cell patch-clamp recordings $\left(V_{\text {hold }}=-60 \mathrm{mV}\right.$ ) on rat hippocampal cells during baseline recordings and $3-5$ min after application of either the C-terminal VGF peptide (TLQP62 ) or N-terminal VGF peptide (LEGS-28). B, TLQP-62 peptides (O) elicited an approximate 1.6-fold increase in synaptic charge within 2 min of exposure (indicated by horizontal bar) which remained elevated over the course of the recording (25 min). LEGS-28 peptides (O), however, did not affect synaptic charge for the duration of treatment. C, Dose-response effect of (-terminal VGF peptides (TLQP-62 and AQEE-30) compared with the response for the $\mathrm{N}$-terminal peptide (LEGS-28). Maximal response is obtained at $0.1 \mu \mathrm{m}$ for both TLQP-62 and AQEE-30. Average synaptic charge 3-5 min after VGF peptide perfusion relative to baseline ( -2 to $0 \mathrm{~min}$ ) is depicted ( $\pm \mathrm{SE}$ ). *Indicates significantly different from LEGS-28 ( $p<0.05$; ANOVA). Number of cells is shown in parentheses. Recordings were obtained from multiple platings.

of VGF on synaptic plasticity, an alternate peptide derived from the C-terminal region was tested (AQEE-30) and revealed a similar effect on synaptic charge (Fig. 5C). The effects of both AQEE-30 and TLQP-62 peptides were dose-dependent, saturating at $0.1 \mu \mathrm{M}$ (Fig. $5 C$ ). Taken together, these data suggest that the functional portion of VGF resides in the C-terminal region of the protein. These findings are the first demonstration of a neuromodulatory role for VGF in synaptic plasticity of the hippocampus that subserves learning and memory.

\section{Discussion}

Here we have shown that BDNF-induced synaptic plasticity is accompanied by transcription of different classes of genes at both $20 \mathrm{~min}$ and $3 \mathrm{hr}$. These genes include IEGs as well as transcripts for ion channels, membrane receptors, neuropeptides, and a phosphatase. This is the first study in which several of these genes have been implicated in BDNF-induced plasticity. Our observations suggest a role for these genes either in the initial phase of synaptic plasticity, in which increased turnover of the molecules is associated with transcription, or in later phases characterized by synaptic strengthening and synaptogenesis. BDNF treatment for such a minimal interval represents a subtle experimental paradigm that affects plasticity and not synaptic morphology. We would therefore not expect large changes in either the magnitude or number of genes expressed. A minimum criterion of 1.7-fold was selected for the original microarrays: after confirmation, the upregulation of these genes was statistically significant.

Among the BDNF-induced genes, several IEGs have previously been implicated in synaptic plasticity and hippocampaldependent learning. For example, Arc is an effector IEG that may directly modulate specific cellular functions. EGR1 and $c$-fos, on 
the other hand, are regulatory transcription factors, which may influence cell function by modulating downstream genes including VGF (Salton et al., 2000; Guzowski et al., 2001). Arc mRNA is rapidly upregulated in response to synaptic activity and is translocated to dendritic segments whose NMDA receptors have been activated (Steward and Worley, 2002). Our findings of increased Arc expression in a hippocampal-dependent task are consonant with studies in which Arc expression correlates with navigation learning (Guzowski et al., 2001). In BDNF-LTP in dentate gyrus, $A r c$ is the most robustly induced of the IEGs, whereas EGR1 is not induced (Ying et al., 2002). In contrast, we find that EGR1 is highly regulated by both BDNF treatment in vitro and training on a hippocampal-dependent task of trace eyeblink conditioning in CA1 and CA3 in vivo. Essential roles for EGR1 (Jones et al., 2001) and $\operatorname{Arc}$ (Guzowski et al., 2000) in maintenance of LTP have recently been described. Our findings that $A r c$ is highly regulated by both acute BDNF treatment in vitro and trace eyeblink conditioning in vivo renders Arc a useful index of transcriptional regulation in response to synaptic activation and perhaps processes of learning dependent on that activation.

\section{Differential synaptic responses}

Using $A r c$ as a prototype, we investigated the phenomenon of differential synaptic responses to BDNF within a population of pyramidal-like hippocampal cells. We found a linear relationship between electrophysiological responses to BDNF and transcriptional changes in single cells, suggesting that altered gene expression contributes to synaptic strengthening. Global synaptic activity was not a predictor of response to BDNF, because there were no differences in basal synaptic activity of responder versus nonresponder populations. The differential synaptic responses to BDNF may therefore be attributable to other factors. The expression of the BDNF receptor, trkB, may vary among neurons. Alternatively, cells derived from the CA1 versus CA3 regions may respond differently to BDNF, which can be examined at the single-cell level using region-specific markers (Zhao et al., 2001). Finally, it is known that hippocampal neuronal responses to BDNF are target-specific; BDNF induces potentiation in excitatory (glutamate) but not inhibitory (GABA) postsynaptic cells (Schinder et al., 2000). However, because we record from pyramidal-like neurons that have been shown to be glutamatergic (Rutherford et al., 1998), differential identity is probably not relevant in our paradigm.

\section{Signal transduction pathways}

Increased gene expression by neurotrophins may provide a mechanism for the maintenance of enhanced synaptic activity. Although the second messenger cascades necessary for trophinmediated synaptic enhancement have been studied, the signal transduction pathways involved in BDNF-induced transcription have not been systematically examined. In neonatal hippocampal slices, BDNF modulation of synaptic plasticity requires activation of MAP kinase and PI3K but not PLC $\gamma$ (Gottschalk et al., 1999), however the PLC $\gamma$ pathway as well as CaMK have been shown to be required for trkB-mediated LTP in adult slices (Minichiello et al., 2002). Thus, differences in the requirement for certain pathways may result from the experimental models used. Other studies have implicated MAP kinase pathways in the induction of BDNF-LTP and indicated that transcription of IEGs is dependent on extracellular signal-regulated kinase (ERK) (Ying et al., 2002). Several other protein kinases, including CaMK, PKA, and PKC have been implicated in the induction and expression of LTP and learning (Adams and Sweatt, 2002; Lisman et al., 2002; Shobe,
2002; Wallenstein et al., 2002). Results shown here indicate that transcription of both IEGs at $20 \mathrm{~min}$ and $V G F$ at $3 \mathrm{hr}$ both require the same signaling pathways. Consequently, common transduction pathways may induce different genes with distinct temporal expression patterns. We found that transcription is specifically dependent on trkB activation. We have also found that MEK, CaMK and PLC $\gamma$ are required for BDNF-induced transcription, whereas PI3K, PKA, and PKC are not. In addition, it has been shown that genes induced by BDNF, including VGF, require CREB activation (Bonni et al., 1995). Our data demonstrate that BDNF-induced transcription uses the same signaling pathways as BDNF-enhanced plasticity, which is consistent with the correlation in Figure 2 between synaptic response to BDNF and Arc induction.

We examined the relevance of transcriptional activation in vitro to changes after memory formation in vivo using the hippocampal-dependent learning task of trace eyeblink conditioning. In addition to increases in IEGs such as $c$-fos, EGR1, and $A r c$, we observed significant upregulation of $V G F$ after learning. These results suggest that the regulation of transcription by $\mathrm{BDNF}$ in vitro can predict changes in vivo. However, several other genes regulated by BDNF in vitro were not significantly induced by eyeblink conditioning, emphasizing the specificity of the regulation in response to associative learning. A number of genes, including BDNF, which are known to be involved in the maintenance of synaptic strengthening, are also upregulated during associative learning (Guzowski et al., 2001; Luo et al., 2001; Cavallaro et al., 2002; Donahue et al., 2002). For instance, during declarative memory formation, of which trace eyeblink conditioning is an example (Clark and Squire, 1998), BDNF expression is enhanced (Tokuyama et al., 2000). In addition, evidence has shown that endogenous BDNF is required for spatial learning and memory (Minichiello et al., 1999; Mu et al., 1999; Alonso et al., 2002; Tyler et al., 2002). Finally, a single nucleotide polymorphism in the targeting region of the human BDNF gene has been linked to deficits in human declarative memory formation (Hariri et al., 2003).

\section{Novel actions of VGF}

Our study is the first to demonstrate VGF mRNA induction after training on an associative learning task, raising the possibility that VGF protein itself may play a functional role in synaptic plasticity associated with learning. We focused on VGF because it is a secreted polypeptide rather than a transcription factor and may potentially mediate neuronal communication. VGF precursor is processed during neuronal differentiation in vitro and becomes localized to large dense core vesicles (Trani et al., 1995; Benson and Salton, 1996; Possenti et al., 1999). Our results demonstrate that VGF transcription is accompanied by translation within $3 \mathrm{hr}$ of BDNF exposure. VGF protein is present in a punctate manner in neurites of hippocampal cultures $12 \mathrm{~d}$ in vitro, suggesting a physiological role for VGF in neuronal modulation.

This report defines a functional role for VGF in the acute regulation of synaptic plasticity. We identified a dose-dependent enhancement of synaptic charge using a synthetic peptide corresponding to the C-terminal region of VGF. These data suggest that a C-terminal cleavage product of precursor VGF is the biologically active species. The excitatory actions of VGF on synaptic transmission and the induction of VGF mRNA after hippocampal-dependent tasks point to a stimulatory role for the peptide in learning and memory. The requirement of BDNF for $V G F$ expression in vivo (Eagleson et al., 2001) and the colocalization of VGF with $\operatorname{trkB}$ (Salton et al., 2000) further implicate VGF 
in synaptic modulation associated with neurotrophins. Consistent with our findings, expression of the peptide is regulated by neuronal activity associated with LTP, monocular deprivation, seizure, and cortical lesions (Hevroni et al., 1998; Snyder et al., 1998). Studies have suggested that VGF may regulate release of other peptides from secretory vesicles (Salton et al., 2000). Therefore, VGF may contribute to synaptic plasticity via enhanced secretion of BDNF or other neuromodulatory peptides in a positive feedback loop. VGF may also mediate the long-term effects of BDNF-induced synaptic strengthening through its ongoing excitatory synaptic actions. The identification of the VGF receptor as well as the analysis of learning in VGF mutant mice (Hahm et al., 1999, 2002) may provide insight into the mechanism of VGF function. Neurotrophins and neuropeptides may therefore act in concert eliciting the long-term maintenance of synaptic plasticity associated with learning and memory.

\section{References}

Adams JP, Sweatt JD (2002) Molecular psychology: roles for the ERK MAP kinase cascade in memory. Annu Rev Pharmacol Toxicol 42:135-163.

Alonso M, Vianna MR, Depino AM, Mello e Souza T, Pereira P, Szapiro G, Viola H, Pitossi F, Izquierdo I, Medina JH (2002) BDNF-triggered events in the rat hippocampus are required for both short- and long-term memory formation. Hippocampus 12:551-560.

Benson DL, Salton SR (1996) Expression and polarization of VGF in developing hippocampal neurons. Brain Res Dev Brain Res 96:219-228.

Berninger B, Schinder AF, Poo MM (1999) Synaptic reliability correlates with reduced susceptibility to synaptic potentiation by brain-derived neurotrophic factor. Learn Mem 6:232-242.

Beylin AV, Gandhi CC, Wood GE, Talk AC, Matzel LD, Shors TJ (2001) The role of the hippocampus in trace conditioning: temporal discontinuity or task difficulty? Neurobiol Learn Mem 76:447-461.

Bonni A, Ginty DD, Dudek H, Greenberg ME (1995) Serine 133phosphorylated CREB induces transcription via a cooperative mechanism that may confer specificity to neurotrophin signals. Mol Cell Neurosci 6:168-183.

Cavallaro S, Dagata V, Alkon DL (2002) Programs of gene expression during the laying down of memory formation as revealed by DNA microarrays. Neurochem Res 27:1201-1207.

Chao MV (2000) Trophic factors: an evolutionary cul-de-sac or door into higher neuronal function? J Neurosci Res 59:353-355.

Chen G, Kolbeck R, Barde YA, Bonhoeffer T, Kossel A (1999) Relative contribution of endogenous neurotrophins in hippocampal long-term potentiation. J Neurosci 19:7983-7990.

Clark RE, Squire LR (1998) Classical conditioning and brain systems: the role of awareness. Science 280:77-81.

Donahue CP, Jensen RV, Ochiishi T, Eisenstein I, Zhao M, Shors T, Kosik KS (2002) Transcriptional profiling reveals regulated genes in the hippocampus during memory formation. Hippocampus 12:821-833.

Eagleson KL, Fairfull LD, Salton SR, Levitt P (2001) Regional differences in neurotrophin availability regulate selective expression of VGF in the developing limbic cortex. J Neurosci 21:9315-9324.

Gottschalk W, Pozzo-Miller LD, Figurov A, Lu B (1998) Presynaptic modulation of synaptic transmission and plasticity by brain-derived neurotrophic factor in the developing hippocampus. J Neurosci 18:6830-6839.

Gottschalk WA, Jiang H, Tartaglia N, Feng L, Figurov A, Lu B (1999) Signaling mechanisms mediating BDNF modulation of synaptic plasticity in the hippocampus. Learn Mem 6:243-256.

Guzowski JF, Lyford GL, Stevenson GD, Houston FP, McGaugh JL, Worley PF, Barnes CA (2000) Inhibition of activity-dependent arc protein expression in the rat hippocampus impairs the maintenance of long-term potentiation and the consolidation of long-term memory. J Neurosci 20:3993-4001.

Guzowski JF, Setlow B, Wagner EK, McGaugh JL (2001) Experiencedependent gene expression in the rat hippocampus after spatial learning: a comparison of the immediate-early genes Arc, c-fos, and zif268. J Neurosci 21:5089-5098.

Hahm S, Mizuno TM, Wu TJ, Wisor JP, Priest CA, Kozak CA, Boozer CN, Peng B, McEvoy RC, Good P, Kelley KA, Takahashi JS, Pintar JE, Roberts JL, Mobbs CV, Salton SR (1999) Targeted deletion of the Vgf gene indi- cates that the encoded secretory peptide precursor plays a novel role in the regulation of energy balance. Neuron 23:537-548.

Hahm S, Fekete C, Mizuno TM, Windsor J, Yan H, Boozer CN, Lee C, Elmquist JK, Lechan RM, Mobbs CV, Salton SR (2002) VGF is required for obesity induced by diet, gold thioglucose treatment, and agouti and is differentially regulated in pro-opiomelanocortin- and neuropeptide Y-containing arcuate neurons in response to fasting. J Neurosci 22:6929-6938

Hariri AR, Goldberg TE, Mattay VS, Kolachana BS, Callicott JH, Egan MF, Weinberger DR (2003) Brain-derived neurotrophic factor val66met polymorphism affects human memory-related hippocampal activity and predicts memory performance. J Neurosci 23:6690-6694.

Hevroni D, Rattner A, Bundman M, Lederfein D, Gabarah A, Mangelus M, Silverman MA, Kedar H, Naor C, Kornuc M, Hanoch T, Seger R, Theill LE, Nedivi E, Richter-Levin G, Citri Y (1998) Hippocampal plasticity involves extensive gene induction and multiple cellular mechanisms. J Mol Neurosci 10:75-98.

Huh GS, Boulanger LM, Du H, Riquelme PA, Brotz TM, Shatz CJ (2000) Functional requirement for class I MHC in CNS development and plasticity. Science 290:2155-2159.

Jones MW, Errington ML, French PJ, Fine A, Bliss TV, Garel S, Charnay P, Bozon B, Laroche S, Davis S (2001) A requirement for the immediate early gene Zif268 in the expression of late LTP and long-term memories. Nat Neurosci 4:289-296.

Korte M, Kang H, Bonhoeffer T, Schuman E (1998) A role for BDNF in the late-phase of hippocampal long-term potentiation. Neuropharmacology 37:553-559.

Lessmann V, Heumann R (1998) Modulation of unitary glutamatergic synapses by neurotrophin- $4 / 5$ or brain-derived neurotrophic factor in hippocampal microcultures: presynaptic enhancement depends on preestablished paired-pulse facilitation. Neuroscience 86:399-413.

Leuner B, Falduto J, Shors TJ (2003) Associative memory formation increases the observation of dendritic spines in the hippocampus. J Neurosci 23:659-665.

Levine ES, Dreyfus CF, Black IB, Plummer MR (1996) Selective role for trkB neurotrophin receptors in rapid modulation of hippocampal synaptic transmission. Brain Res Mol Brain Res 38:300-303.

Lisman J, Schulman H, Cline H (2002) The molecular basis of CaMKII function in synaptic and behavioural memory. Nat Rev Neurosci 3:175-190.

Lu B (2003) BDNF and activity-dependent synaptic modulation. Learn Mem 10:86-98.

Luo Y, Long JM, Spangler EL, Longo DL, Ingram DK, Weng NP (2001) Identification of maze learning-associated genes in rat hippocampus by cDNA microarray. J Mol Neurosci 17:397-404.

Messaoudi E, Ying SW, Kanhema T, Croll SD, Bramham CR (2002) Brainderived neurotrophic factor triggers transcription-dependent, late phase long-term potentiation in vivo. J Neurosci 22:7453-7461.

Minichiello L, Korte M, Wolfer D, Kuhn R, Unsicker K, Cestari V, RossiArnaud C, Lipp HP, Bonhoeffer T, Klein R (1999) Essential role for TrkB receptors in hippocampus-mediated learning. Neuron 24:401-414.

Minichiello L, Calella AM, Medina DL, Bonhoeffer T, Klein R, Korte M (2002) Mechanism of TrkB-mediated hippocampal long-term potentiation. Neuron 36:121-137.

Mizuno M, Yamada K, Takei N, Tran MH, He J, Nakajima A, Nawa H, Nabeshima T (2003) Phosphatidylinositol 3-kinase: a molecule mediating BDNF-dependent spatial memory formation. Mol Psychiatry 8:217-224.

Mu JS, Li WP, Yao ZB, Zhou XF (1999) Deprivation of endogenous brainderived neurotrophic factor results in impairment of spatial learning and memory in adult rats. Brain Res 835:259-265.

Nguyen PV, Abel T, Kandel ER (1994) Requirement of a critical period of transcription for induction of a late phase of LTP. Science 265:1104-1107.

Patterson SL, Pittenger C, Morozov A, Martin KC, Scanlin H, Drake C, Kandel ER (2001) Some forms of cAMP-mediated long-lasting potentiation are associated with release of BDNF and nuclear translocation of phospho-MAP kinase. Neuron 32:123-140.

Phillips J, Eberwine JH (1996) Antisense RNA amplification: a linear amplification method for analyzing the mRNA population from single living cells. Methods 10:283-288.

Poo MM (2001) Neurotrophins as synaptic modulators. Nat Rev Neurosci 2:24-32. 
Possenti R, Rinaldi AM, Ferri GL, Borboni P, Trani E, Levi A (1999) Expression, processing, and secretion of the neuroendocrine VGF peptides by INS-1 cells. Endocrinology 140:3727-3735.

Rutherford LC, Nelson SB, Turrigiano GG (1998) BDNF has opposite effects on the quantal amplitude of pyramidal neuron and interneuron excitatory synapses. Neuron 21:521-530.

Salton SR, Ferri GL, Hahm S, Snyder SE, Wilson AJ, Possenti R, Levi A (2000) VGF: a novel role for this neuronal and neuroendocrine polypeptide in the regulation of energy balance. Front Neuroendocrinol 21:199-219.

Schinder AF, Berninger B, Poo M (2000) Postsynaptic target specificity of neurotrophin-induced presynaptic potentiation. Neuron 25:151-163.

Shobe J (2002) The role of PKA, CaMKII, and PKC in avoidance conditioning: permissive or instructive? Neurobiol Learn Mem 77:291-312.

Snyder SE, Cheng HW, Murray KD, Isackson PJ, McNeill TH, Salton SR (1998) The messenger RNA encoding VGF, a neuronal peptide precursor, is rapidly regulated in the rat central nervous system by neuronal activity, seizure and lesion. Neuroscience 82:7-19.

Steward O, Worley P (2002) Local synthesis of proteins at synaptic sites on dendrites: role in synaptic plasticity and memory consolidation? Neurobiol Learn Mem 78:508-527.

Suen PC, Wu K, Levine ES, Mount HT, Xu JL, Lin SY, Black IB (1997) Brain-derived neurotrophic factor rapidly enhances phosphorylation of the postsynaptic $N$-methyl-D-aspartate receptor subunit 1. Proc Natl Acad Sci USA 94:8191-8195.

Thakker-Varia S, Alder J, Crozier RA, Plummer MR, Black IB (2001) Rab3A is required for brain-derived neurotrophic factor-induced synaptic plas- ticity: transcriptional analysis at the population and single-cell levels. J Neurosci 21:6782-6790.

Thoenen H (2000) Neurotrophins and activity-dependent plasticity. Prog Brain Res 128:183-191.

Tokuyama W, Okuno H, Hashimoto T, Xin Li Y, Miyashita Y (2000) BDNF upregulation during declarative memory formation in monkey inferior temporal cortex. Nat Neurosci 3:1134-1142.

Trani E, Ciotti T, Rinaldi AM, Canu N, Ferri GL, Levi A, Possenti R (1995) Tissue-specific processing of the neuroendocrine protein VGF. J Neurochem 65:2441-2449.

Tyler WJ, Alonso M, Bramham CR, Pozzo-Miller LD (2002) From acquisition to consolidation: on the role of brain-derived neurotrophic factor signaling in hippocampal-dependent learning. Learn Mem 9:224-237.

Wallenstein GV, Vago DR, Walberer AM (2002) Time-dependent involvement of PKA/PKC in contextual memory consolidation. Behav Brain Res 133:159-164.

Yamada K, Mizuno M, Nabeshima T (2002) Role for brain-derived neurotrophic factor in learning and memory. Life Sci 70:735-744.

Ying SW, Futter M, Rosenblum K, Webber MJ, Hunt SP, Bliss TV, Bramham CR (2002) Brain-derived neurotrophic factor induces long-term potentiation in intact adult hippocampus: requirement for ERK activation coupled to CREB and upregulation of Arc synthesis. J Neurosci 22:1532-1540.

Zhao X, Lein ES, He A, Smith SC, Aston C, Gage FH (2001) Transcriptional profiling reveals strict boundaries between hippocampal subregions. J Comp Neurol 441:187-196. 